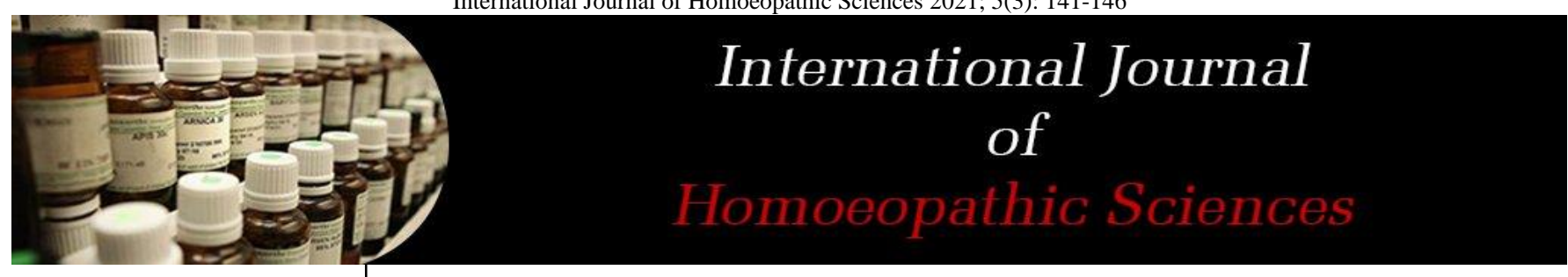

E-ISSN: 2616-4493 P-ISSN: 2616-4485 www.homoeopathicjournal.com IJHS 2021; 5(3): 141-146 Received: 13-05-2021 Accepted: 15-06-2021

Dr. Mithilesh Chandra Suri Associate Professor \& HOD, Dept. of Pathology \& Microbiology Govt.

Homoeopathic Medical College \& Hospital, Godda,

Jharkhand, India
Corresponding Author: Dr. Mithilesh Chandra Suri Associate Professor \& HOD, Dept. of Pathology \& Microbiology Govt. Homoeopathic Medical College \& Hospital, Godda, Jharkhand, India

\section{An overview of frozen shoulder with homoeopathic management}

\section{Dr. Mithilesh Chandra Suri}

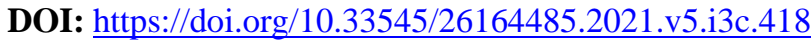

Abstract

Frozen shoulder is very common suffering among the all joint disorder. It is more common in diabetes mellitus patient. About 77 million people suffering from diabetes mellitus in India. The global diabetes prevalence in 2019 is estimated to be $9.3 \%$ (463 million people), rising to $10.2 \%$ (578 million) by 2030 and $10.9 \%$ ( 700 million) by 2045 . As the diabetes suffering increases the frequency of frozen shoulder cases will also increase. Since the developing country like India \& other South Asian Countries can't afford the expansive surgical intervention. Homoeopathy can deals with the situation in cost-effective, simple and reliable way and can serve the humanity through medicine along with recommended exercises. Thus, taking an overview on frozen shoulder with its homoeopathic treatment and managements is the need of time.

Keywords: frozen shoulder, diagnosis, clinical features, homoeopathy, exercise

\section{Introduction}

Frozen Shoulder (Syn: Periarthritis, Adhesive Capsulitis): It is defined as a clinical syndrome characterized by painful restriction (Figs A and B) of both active and passive shoulder movements due to causes within the shoulder joint or remote (other parts of the body) ${ }^{[1]}$

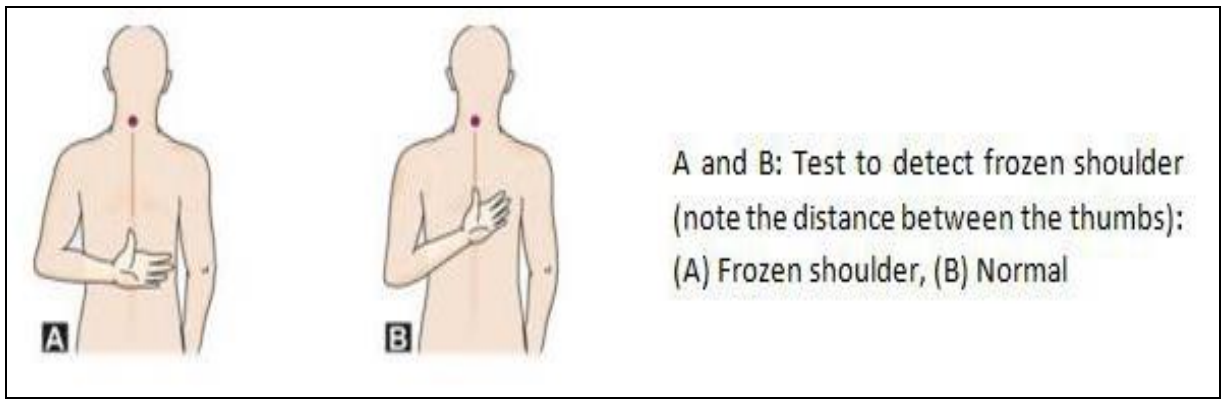

Epidemiology and Background

Dupley first described it in 1872 and called it as humero-scapular periarthritis. In 1934, Codman coined the term Frozen shoulder, and in 1945, Neviaser gave the name adhesive capsulitis. "frozen shoulder," adhesive capsulitis is commonly encountered in the outpatient setting, the prevalence of adhesive capsulitis ranges from $2 \%$ to $5 \%$ but can be as high as $30 \%$ in patients with insulin dependent diabetes mellitus (IDDM). Bilateral: 12\%. Comorbid IDDM is associated with a worse prognosis and increased likelihood for surgical intervention. While the exact pathogenesis remains unclear, other factors associated with adhesive capsulitis include female sex, age over 40 years ${ }^{[2]}$.

\section{Causes}

The causes for frozen shoulder could be: ${ }^{[1]}$

- Primary: Here the exact cause is not known and it could be idiopathic.

- Secondary: According to Lumberg, the secondary causes could be:

- Shoulder causes: Shoulder joint which can give rise to frozen shoulder are tendonitis of rotator cuff, bicipital tendinitis, fractures and dislocations around the shoulder, etc.

- Nonshoulder causes: Problems like diabetes, cardiovascular diseases with referred 
pain to the shoulder, which keeps the joint immobile, reflex sympathetic dystrophy, frozen hand shoulder syndrome, a complication of Colles' fracture, can all lead to frozen shoulder. The reason could be prolonged immobilization of the shoulder joint due to referred pain, etc.

\section{Pathology}

- During abduction, and repeated overhead activities of the shoulder, long head of biceps and rotator cuff undergo repeated strain. This results in inflammation, fibrosis and consequent thickening of the shoulder capsule and adhesion to the humeral neck. As a result, there is decreased synovial fluid within the joint with diminished overall joint volume., which results in loss of movements (Figs: A and B). If the movements are continued, then the fibrosis gradually breaks, movements return but never come back to normal ${ }^{[1]}$.

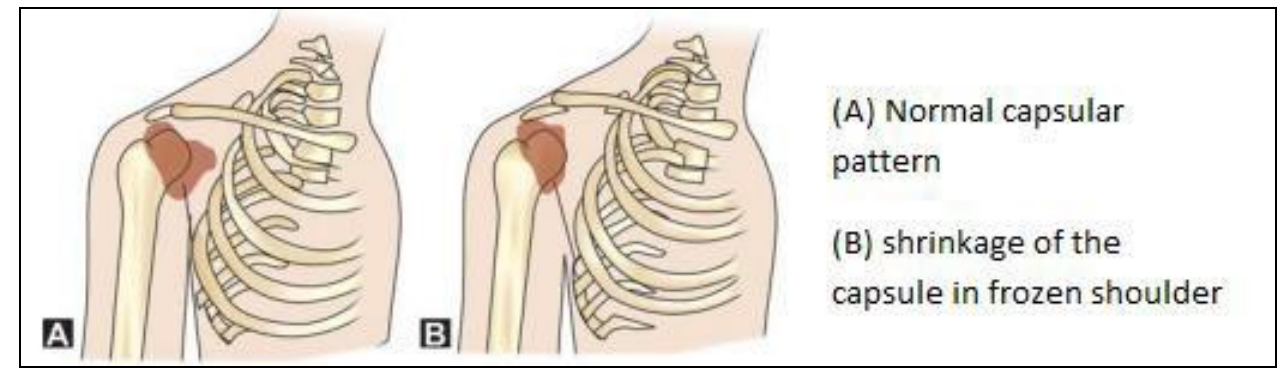

- Prolonged activity causes small scapular and biceps muscles to waste faster, load on joint increases and degenerative changes sets in. Capsule is fibrosed and shoulder movements are decreased.

\section{Clinical Features}

Adhesive capsulitis is characterized by an insidious onset of shoulder pain for several months with a global limitation of both active and passive range of motion.

A patient with frozen shoulder clinically presents as follows:

- Decreased range of both active and passive shoulder movements.

- The patient demonstrates a capsular pattern of movement restrictions (i.e. external rotation > abduction $>$ internal rotation).

- Pain is noted at the end stage of stretch. Accessory joint play is reduced.

- Resistive tests are generally pain free in the available range of motion.

- Patient is unable to do routine daily activities like combing the hair, in case of women wearing the buttons of their blouse, (Fig.), doing overhead activities, etc. ${ }^{[1]}$

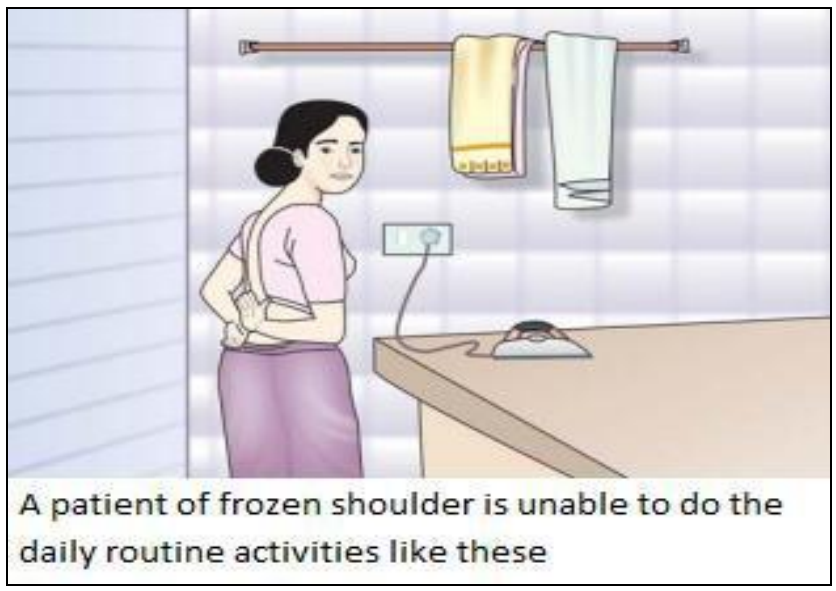

\section{Clinical Stages}

There are four classical stages in frozen shoulder, according to Reeves [2]:

Stage I: the pre-adhesive stage, is characterized by a fibrinous inflammatory synovitic reaction without adhesion formation. As such, patients usually have full motion but have pain, often at night. Misdiagnosis is common at this stage.

- Stage II (stage of pain): progresses to an acute synovitis with synovial proliferation and early adhesion formation. Pain is prominent, but loss of motion remains mild. This stage lasts for 10- 36 weeks.

Note: Many of the literature consider Stage I and II in a single stage.

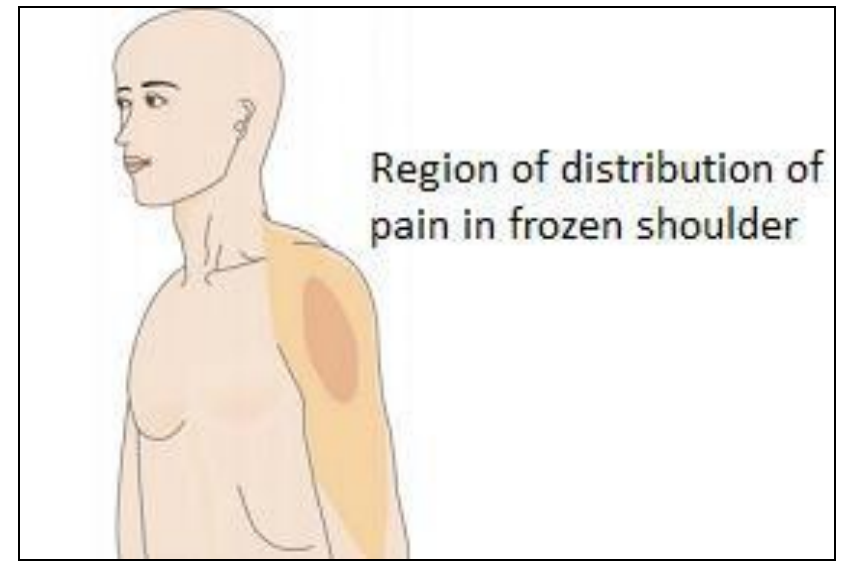

- Stage III (stage of stiffness): Pain gradually decreases and the patient complains of stiff shoulder. It referred as the maturation stage wherein inflammation and hence pain have decreased; however, more fibrosis is present, and range of motion becomes further limited. Slight movements are present. This lasts for 4-12 months.

Stage IV (stage of recovery): Patient will have no pain and movements would have recovered but will never be regained to normal. Chronic stage is characterized by adhesions that have matured resulting in severely reduced motion. It lasts for 6 months to 2 years.

\section{Miasmatic Analysis}

Clinical stage I \& II is directly related to the inflammatory lesion which is psoric in nature and predominantly associate with pain, loss of motion/decreased movement of shoulder joint. The stage of pain with inflammation progressively developed in sycotic miasm. 
Stage III is predominantly sycotic with some extent of psoric manifestation with restricted movement, stiffness, chronic inflammation and stage of fibrosis. Gradually in stage IV the pain subside and permanent fibrosis and stiffness permanently established in shoulder joint and it complete the sycotic phase.

\section{Diagnosis}

During the physical exam, move in certain ways to check for pain and evaluate the range of motion (active range of motion). Usually it diagnosed from signs and symptoms. Xrays or an MRI - to rule out other problems ${ }^{[1]}$.

\section{- Radiology}
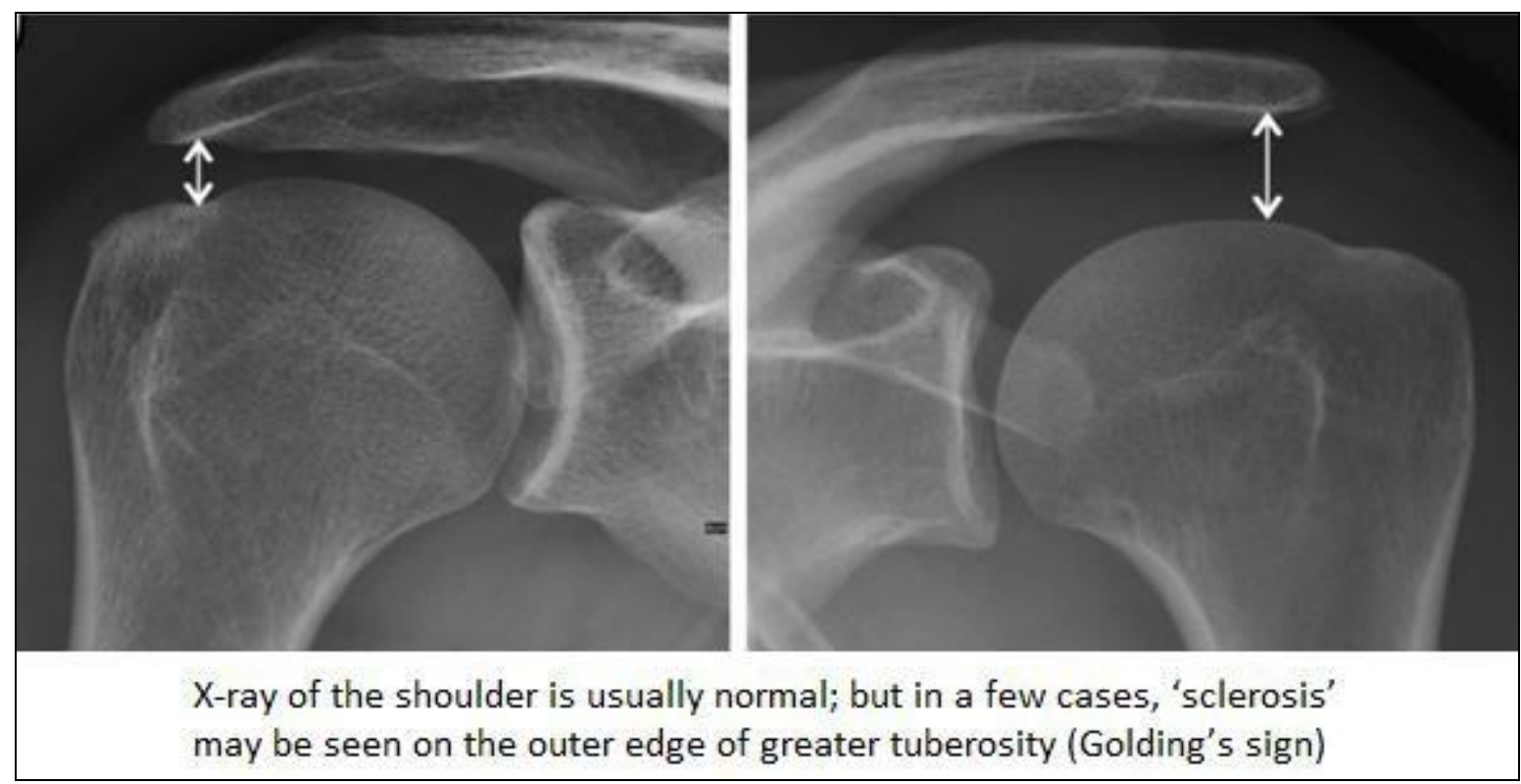

\section{Differential Diagnosis}

The differential diagnosis includes:

- Acromioclavicular arthropathy,

- Autoimmune disease,

- Biceps tendinopathy,

- Glenohumeral osteoarthritis,

- Dotator cuff tendinopathy or tear,

- Subacromial and subdeltoid bursitis

- TREATMENT \& MANAGEMENT

- Stage I\&II: Management of pain and recommended exercises.

- Stage III: In this stage, since the pain will have reduced considerably, exercises both active and passive are gradually begun followed by physiotherapy, ultrasound, heat and shoulder wheel exercises.

- Stage IV: In this stage, active and passive exercises, physiotherapy consisting of short wave diathermy, ultrasound, etc. are continued.

\section{Some Reportorial Rubrics from Kent's Repertory ${ }^{[4]}$}

- BACK p. 903 PAIN, dorsal region, scapulæ, left, under, cough, during, hang down, arm :

- Extremities p. 1009

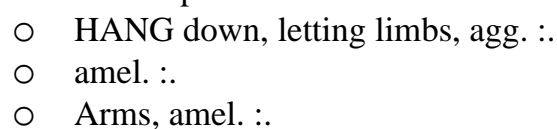

- EXTREMITIES p. 1018, INFLAMMATION, Joints.

O Tendons :

○ Upper limbs :

- Extremities, lameness, (p. 1031-32) Shoulder : O shoulder, right :

- EXTREMITIES p. 1037 NUMBNESS, upper limbs,

O Shoulder :

O Upper arm:

○ deltoid region:
- EXTREMITIES p. 1047 PAIN, attachment of muscles Joints:

- EXTREMITIES p. 1051-52 PAIN, upper limbs, Shoulder :

O right :

$\bigcirc$ then left :

O left :

- dislocation, as of :

- Extremities p. 1055 PAIN, upper arm,
○ deltoid region
0 right
○ left

- EXTREMITIES p. 1103 PAIN, drawing, upper arm; deltoid region

- EXTREMITIES p. 1160 PAIN, tearing, upper arm; deltoid region

- EXTREMITIES p. 1191, STIFFNESS :

O Joints :

O Shoulder : right :

- Extremities p. 1197 SWELLING, upper limbs, paralysis, after, Shoulder

Homoeopathic Medicine ${ }^{[3,5]}$

Calcarea phos: Frozen shoulder where pain gets worse from any change of weather. Pains start from left then to right and may travel downward to the arm similarly. Feels complaints more when thinking about them. There's pain and stiffness in shoulders and shoulder-blades. Stiffness and pain with a cold feeling, worse change of weather. Crawling and coldness.

Ferrum met: Left-sided frozen shoulder. Shootings and tearings in the joint of the shoulder, and in the arm. Pain is of drawing, shooting, tearing and laming character. Paralytic tearing pain from the shoulder joint into the upper left arm 
and patient may feel it impossible to raise the arm. Cracking in shoulder joints. Aggravation of pain by motion. Pains get better by warmth. Worse while sweating, sitting still. After cold washing and overheating. Midnight aggravation.

Ferrum phos: Shoulder which gets relieved by moving the arm gently. Stiff neck, pain extended to chest and wrist. Palm hot, hand swollen and painful. Acute rheumatism of right deltoid. Joint red, swollen, very sensitive. First stage of inflammation. Shoulder is stiff, painful and highly sensitive to touch. Violent tearing pain right shoulder and upper arm. Worse, at night and 4 to 6am, touch, jar, motion. Better cold application.

Rhus. Tox: Painful tension between shoulder blades. Pain in the shoulders and back, as from a sprain. Tearing and burning pain in shoulder, with lameness in the arm. Hot painful swelling of joints. Pain along the ulnar nerve. Loss of power in fore arm and fingers. Stiffness in the nape of neck, Pain between the shoulders on swallowing. Aliment from; spraining or straining a single part, muscle or tendon, over lifting. Worse during sleep, cold, wet rainy weather, when lying on the back or in the right side. Better, warmth, dry weather, motion, rubbing.

Ruta g.: Dull tearing in bones of the arm and joints of elbow. Pain in forearms as well as in bones and joints of hands as if they had been beaten. Bruised lame sensation all over, as agter a fall or blow; worse in limbs and joints.
Restless, turns and changes position frequently when lying. Lameness after sprains. Pain in the nape, back and loins. Backache better pressure and lying on back.

Sanguinaria can: Pains are present in the top of right shoulder, arm and nape of neck. Difficulty in raising the arm. Right side neuritis Pains usually get worse during the night and while attempting to turn in bed. The patient feels great difficulty to raise the arm on account of distressing pains. Rheumatic pain in right arm and shoulder worse at night in bed, cannot raise arm, movements cause excessive pain. Pain in right deltoid.

Guaiacum Officinale: Immovable stiffness of joints. Pain from the head to neck. Aching in the nape. Stiff neck and sore shoulder. Stitches between the scapulae. Contractive pain between scapulae. There is a sharp stitching type of pains in the top of the shoulder. Pains are of drawing and lacerating nature which can travel down the arm. Worse from motion, heat, pressure.

\section{Recommended Exercises}

Always warm up the shoulder before performing the frozen shoulder exercises. the most effective way to try this is to require a warm shower or bath for 10 to fifteen minutes. Stretch to the point of tension but not pain. Perform these all exercise 10 to 20 times a day ${ }^{[6]}$.

\section{Pendulum stretch}

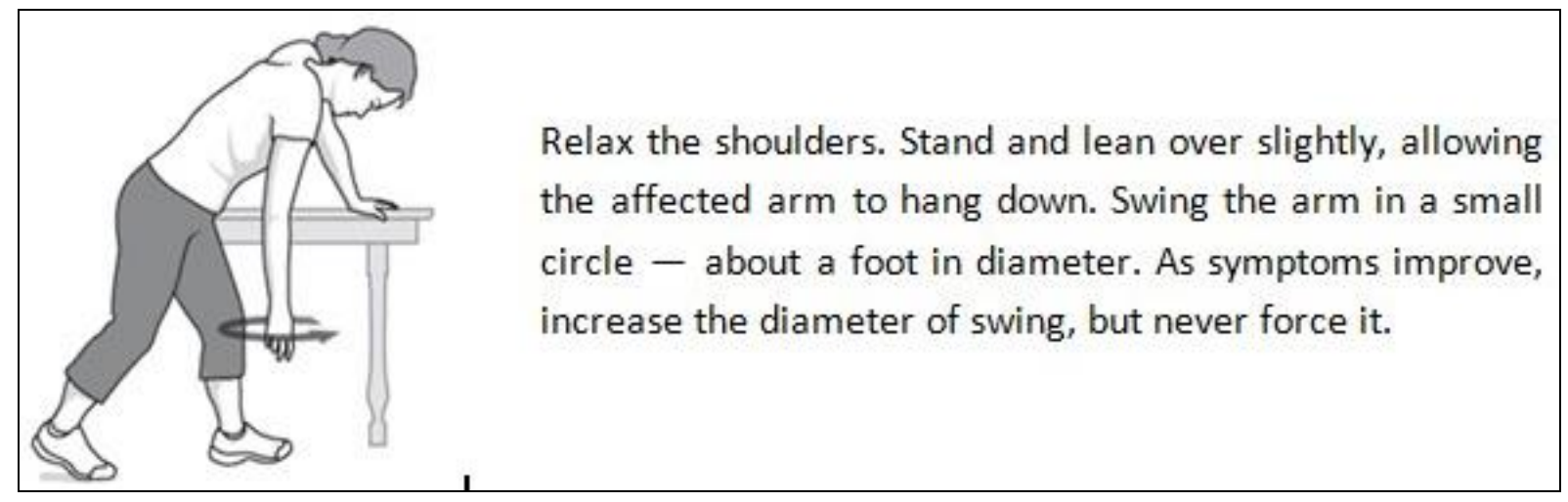

\section{Towel stretch}

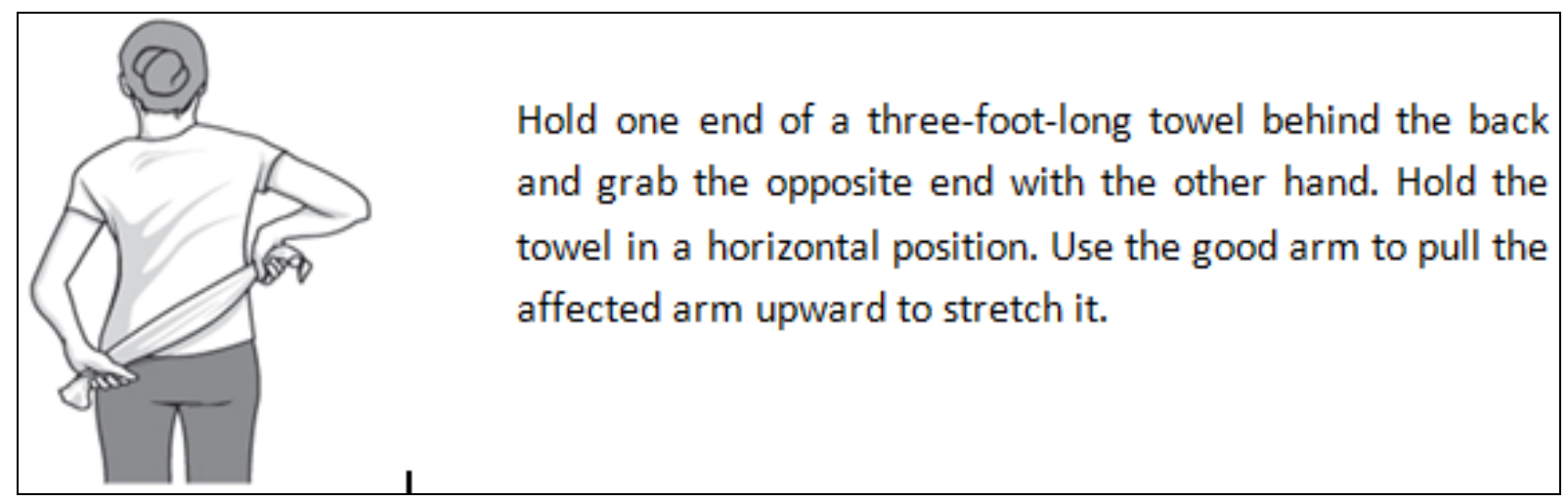




\section{Finger walk}

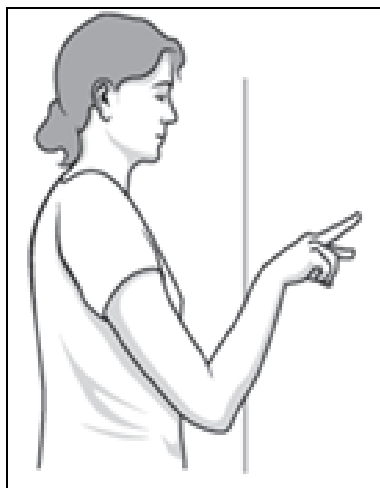

Face wall three-quarters of an arm's length away. Reach out and touch the wall at waist level with the fingertips of the affected arm. With the elbow slightly bent, slowly walk the fingers up the wall, spider-like, until raised the arm as far as comfortably.

4. Cross-body reach

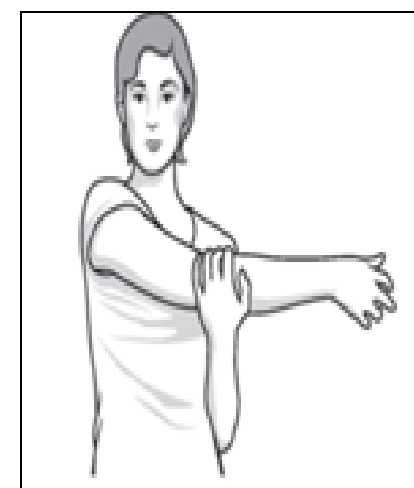

Sit or stand. Use the good arm to lift the affected arm at the elbow, and bring it up and across the body, exerting gentle pressure to stretch the shoulder. Hold the position for 15 to 20 seconds.

\section{Armpit stretch}

Using the good arm, lift the affected arm onto about
breast-high. Gently bend the knees, opening up the
armpit. Deepen the knee bend slightly, gently stretching
the armpit, and then straighten. With each knee bend,
stretch a little further, but don't force it.

6. Outward rotation

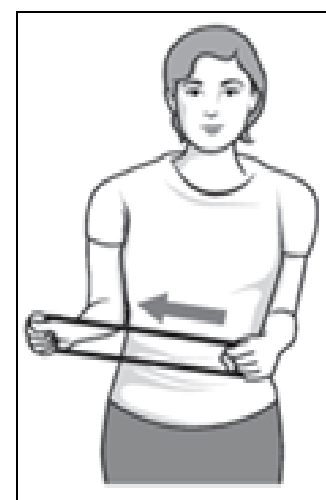

Hold a rubber exercise band between the hands with the elbows at a 90-degree angle close to the sides. Rotate the lower part of the affected arm outward two or three inches and hold for five seconds. 


\section{Inward rotation}

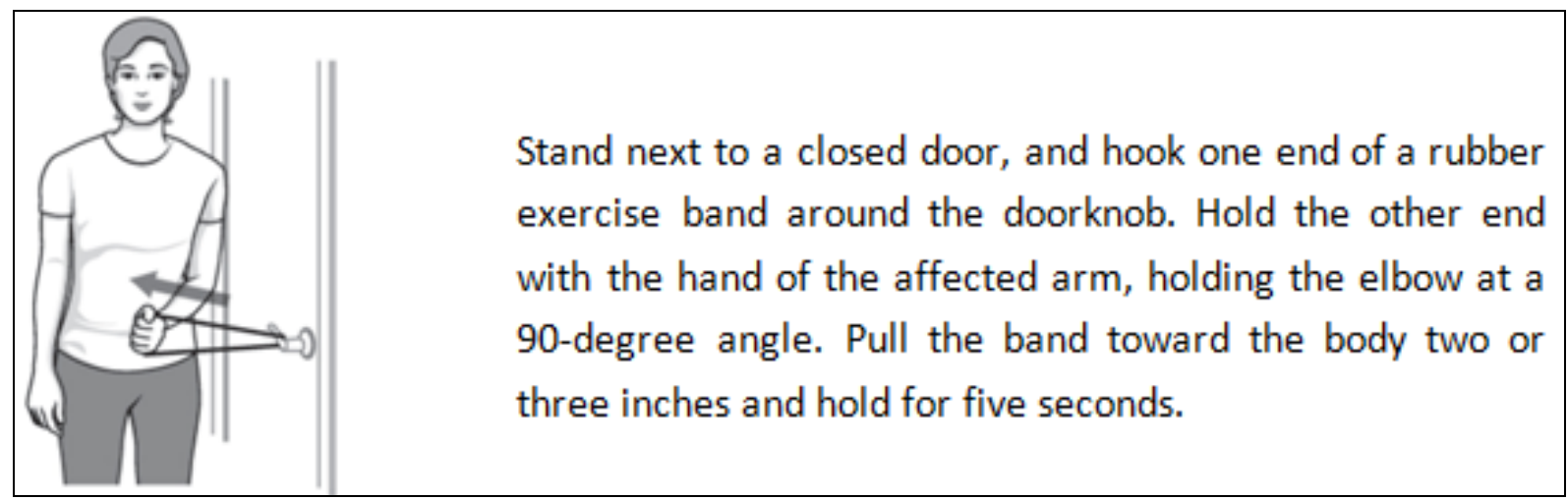

\section{Conclusion}

The right choice of medicines (Remedy) and correct exercise based on different stages can be very effective in the treatment of frozen shoulder. Correct diagnosis, miasmatic analysis and therapeutic approach will be helpful in this.

\section{References}

1. Ebnezar John, Textbook of Orthopedics: Regional Conditions of the Upper Limb, 4th Edition, New Delhi, Jaypee Brothers Medical Publishers (P) Ltd, 2010.

2. Katz Jeffrey N, Blauwet Cheri A, Schoenfeld Andrew J. Principles of Orthopedic Practice for Primary Care Providers: Shoulder Soft Tissue Pathology, Switzerland, Springer International Publishing 2018.

3. Boericke W. New Manual of Homoeopathic Materia Medica with Repertory. Third Revised and Augmented Edition. New Delhi: B. Jain Publishers Pvt. Ltd 2007.

4. Kent JT. Repertory of the Homoeopathic Materia Medica, 6th American Edition, New Delhi, B. Jain Publishers (P) ltd 2006.

5. Allen HC. Keynotes Rearranged and Classified with Leading Remedies of the Materia Medica and Bowel Nosodes. 8th edition. New Delhi: B. Jain Publishers Pvt. Ltd 2018.

6. https://www.health.harvard.edu/shoulders/stretchingexercises-frozen-shoulder 